\title{
DISCUSSION PAPER: PRACTICAL METHODS
}

\author{
Daniel T. Snyder \\ The University of Michigan School of Dentistry \\ Ann Arbor, Michigan 48104
}

The objective of this discussion following three fine presentations is to help direct our consideration as professionals to these and other practical methods of improving the health and happiness of hemophiliacs. This will require a great deal of cooperative effort and dedication by physicians and dentists across these United States in the same manner demonstrated by the participants of this symposium. It will require distinct behavorial changes by health care professionals whose responsibility it is to render the care. It will likewise require distinct behavorial changes in the population of hemophiliac families who are so desperately in need of routine dental health care.

Moss proclaimed the keynote to optimum dental health for hemophiliacs when he discussed preventive dentistry. This is the practical solution to the problems of disease, pain, inconvenience, and devastating costs that result from neglect of oral health care. In addition to the preventive measures that he mentioned, it is appropriate to emphasize the area of fluoridation. Community water fluoridation, dietary supplements, topical applications, and fluoridecontaining dentifrices all contribute to effective prevention or reduction of dental caries.

One of his suggestions seemed a bit unjustified. The removal of primary teeth as much as a year prematurely as a matter of convenience during treatment for other bleeding episodes seems rather unnecessary. Our experience has indicated that relatively few serious problems arise in association with exfoliation of primary teeth in hemophilic patients. Aggressive restorative treatment and preventive maintenance are necessary to avoid complications and risks.

Lucas reiterated the importance of preventive dentistry for hemophiliacs. He has dedicated years to improving surgical methods and to the preparation of the hemophiliac to receive surgical care. Until a greater number of dentists become well trained in the use of hypnosis, however, dental treatment can be performed effectively under the influence of local anesthetics and nitrous oxide-oxygen analgesia. More clinical study is needed to develop simpler methods of complete and effective control of pain.

Walsh's report has brought us up to date on the use of epsilon-aminocaproic acid (EACA) therapy for dental extractions. His effort and recent survey confirms his previous study as well as those recently reported by Webster and colleagues $^{1}$ and Corrigan. ${ }^{2}$ At the University of Michigan Medical Center, this mode of therapy for dental extractions has been directed by J. A. Penner in cooperation with personnel in the Department of Dentistry utilizing an EACA dosage of $40 \mathrm{mg} / \mathrm{kg}$ within one hour prior to and at four-hour intervals postoperatively, and was based on a study by Megquier ${ }^{3}$ that identified excessive fibrinolytic activity in the extraction site immediately following surgery. With this regimen having become established it is appropriate to consider its use as a supportive measure for extensive periodontal therapy as outlined by 
Webster and Courtney. ${ }^{*}$ Its use with outpatient therapy would mean a reduction of stress on the patient as suggested by Agle ${ }^{5}$ and substantial savings in time and cost.

Thus far our attention has been focused upon treatment philosophies, techniques, and their development and use. In recent years several articles ${ }^{6-8}$ have provided rational approaches to modern dental treatment for hemophiliacs. Now it is time to stress implementation and delivery of quality dental services to the hemophilic population. That objective has been pursued in Michigan with a program that deserves a brief description.

Throughout the state of Michigan there are more than 1000 known individuals with hemorrhagic disorders. FIGURE 1 shows the distribution of these persons by counties. They have access to any of the specialized hospital treatment centers, which are strategically located, as shown in FIgURE 2 . The treatment centers together are staffed by more than 25 hematologists and pediatricians.

Five years ago Hemophilia of Michigan, in cooperation with John Penner, Director of Hemophilia Control at the University of Michigan Medical Center, developed a program for hemophilia identification as a means to improve services for individuals with hemorrhagic disease problems and also permit expansion of professional education. The program was implemented by conducting one-day field clinics at various locations throughout the state. To date these clinics have been held one or more times at Ann Arbor, Marquette, Grand Rapids, Detroit, Flint, Traverse City, and Lansing. These locations were selected because they provided adequate hospital facilities and qualified personnel and were readily accessible to families within a radius of five to 100 miles.

\section{Field Clinics}

The personnel for each diagnostic and evaluation clinic activity were derived from two sources: (1) a core staff from the University of Michigan Medical Center of a hematologist, orthopedist or doctor of physical medicine, dentist, medical technician, nurse specialist (hematology), and social worker, and (2) their counterparts if available from the community. The functions performed at each clinic activity included laboratory studies, general medical and orthopedic examinations, dental evaluations, and counseling services.

The dental evaluations were conducted at the hospital site whenever facilities were available to perform an adequate visual screening and $X$-ray examination of the oral structures. If inadequate facilities existed, arrangements were made for the cooperative use of the private office of a nearby local dental practitioner. Together the visiting staff dentist and the local practicing dentist examined and evaluated the oral health condition of each patient. The findings were recorded on a written form as illustrated in FIGURE 3. Posterior bitewing radiographs and selected periapical radiographs were taken for each patient. These were processed on site whenever possible or upon return to Ann Arbor. In addition to information about the teeth, findings for the oral soft tissues were also recorded.

As soon as radiographic findings were read and recorded, appropriate treatment recommendations were listed. A copy of the completed information form and the radiographs were mailed to the family dentist with a brief statement 


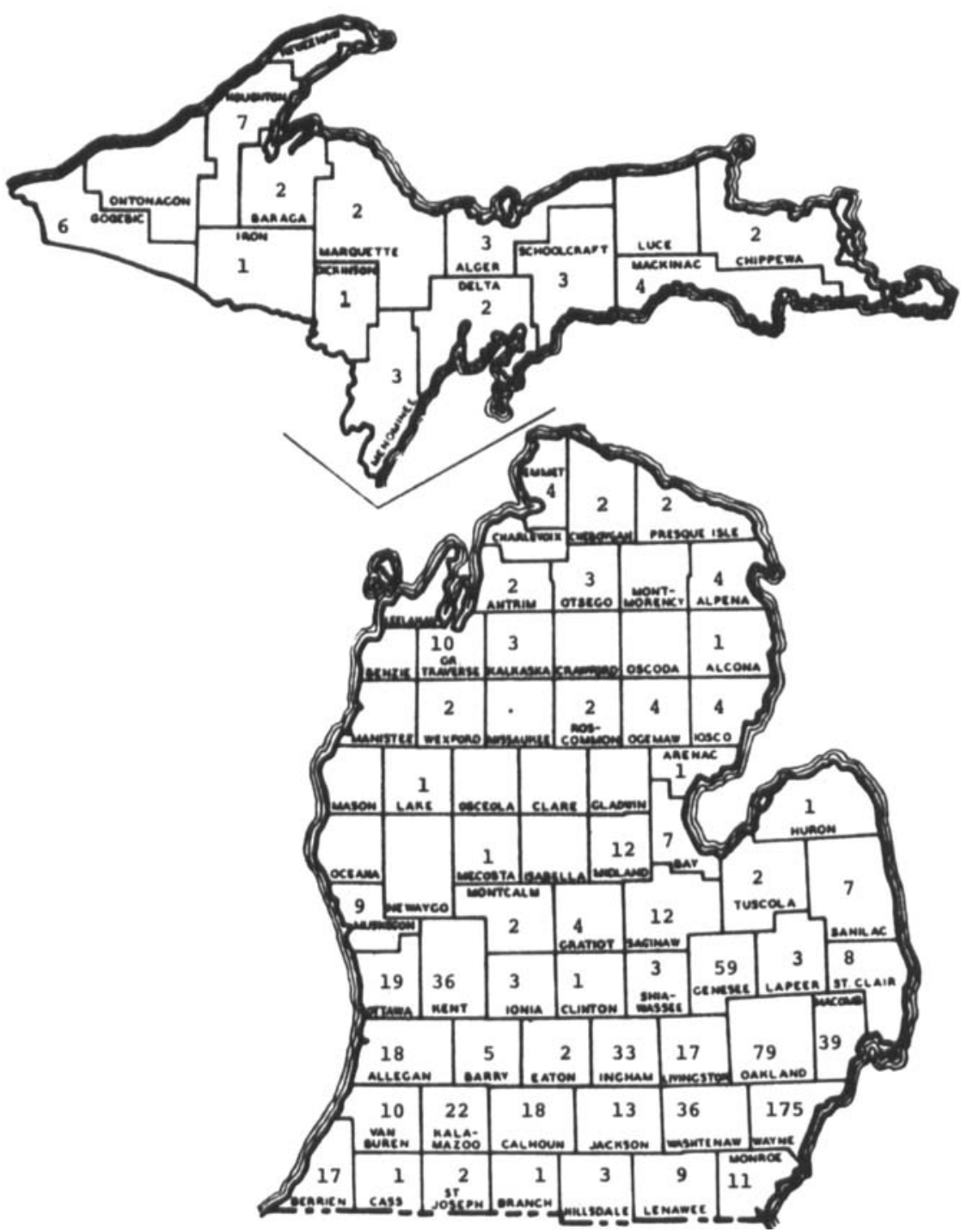

FIGURE 1. Known hemophiliacs by Michigan county. 


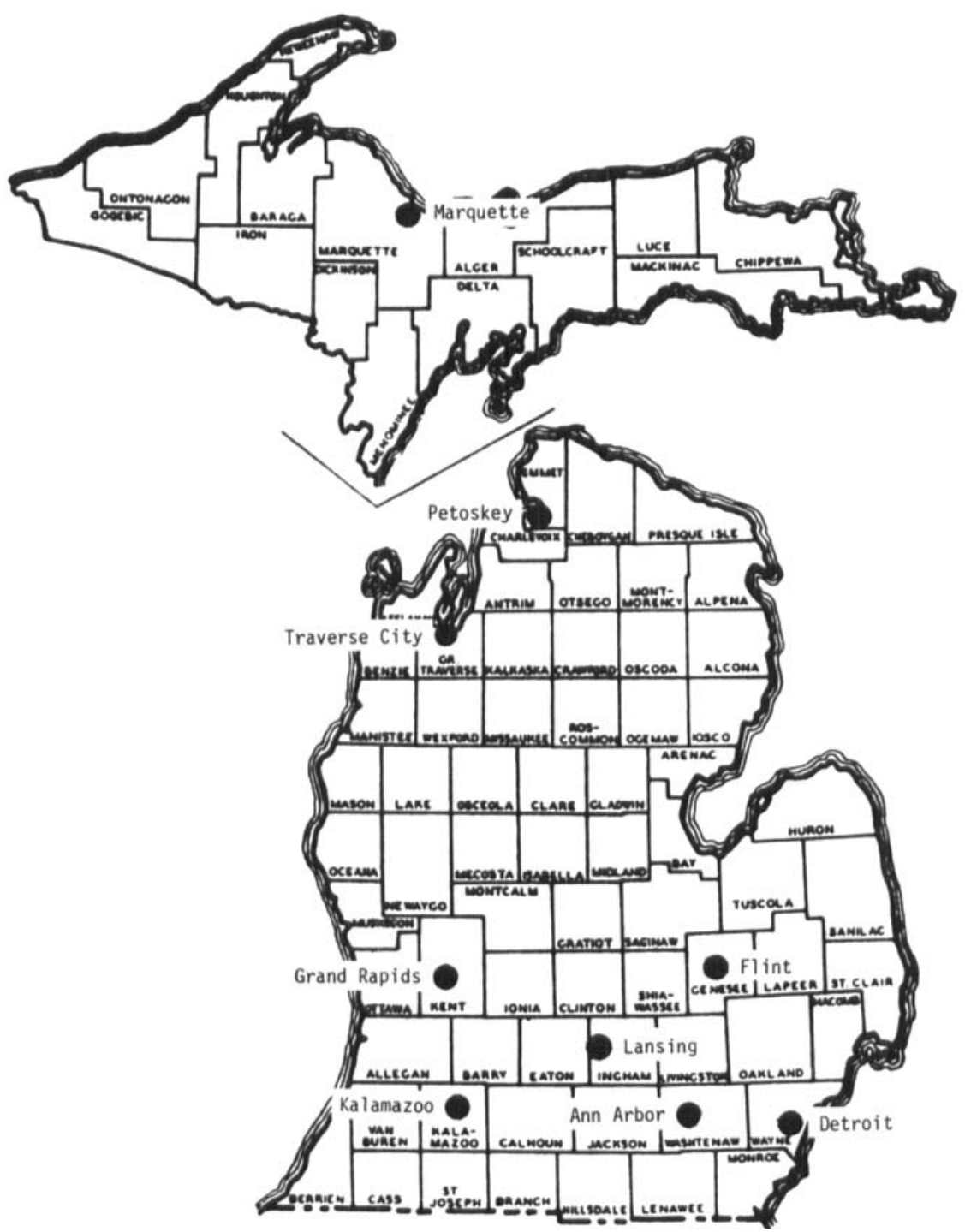

FIGURE 2. Michigan institutions where treatment of hemophiliacs is available.

explaining the clinic activity. During the clinic session informal discussions were held with the patients and parents urging improvement in their home oral health maintenance and that they visit their family dentist regularly. Problems such as lack of transportation or inadequate funds to obtain dental treatment were discussed with the social worker, and if necessary the patient was directed to the appropriate agency for aid.

The local practicing dentists who have participated in the diagnostic and evaluation clinics have been most willing and cooperative. This activity has 
provided an excellent forum for discussion and exchange of information about the feasibility and advantages of treating hemophiliacs in the private office.

A continuing education activity also was coordinated with the field clinics. Local physicians and dentists were invited to meet for a luncheon at the participating hospital facility. Brief presentations were given by the visiting staff regarding diagnosis and treatment of hemophilic patients. Opportunity was given for the local professionals to discuss questions and problems they may have experienced when treating hemophilic patients.

The oral health conditions of the persons examined at the field clinics were highly variable. Findings indicated that many persons with hemophilia had obtained regular dental care and had managed their home care satisfactorily. However, some individuals exhibited a deplorable oral condition that increased

HMWOPHILIA DIAGNOSTIC AND EVALUATION CLINIC DENTAL EXAMIRATION

Date $12-15-75$

Ann Ardior

\begin{tabular}{|c|c|c|c|c|c|c|c|c|c|c|c|c|c|c|c|c|}
\hline$\pi$ & $x$ & $x^{2}$ & 3 & $y_{A}$ & ${ }_{B}$ & {$\left[\begin{array}{l}c \\
\end{array}\right.$} & 78 & ${ }^{8}$ & $y^{9}$ & $y^{10}$ & ${ }_{17}$ & $I^{2}$ & $\begin{array}{l}75 \\
3\end{array}$ & 14 & $\bar{X}$ & ${ }^{18}$ \\
\hline $\mathrm{R}$ & & & 0 & & & & & & & & & & & & & \\
\hline $\mathrm{Cc}$ & & & & & & & & & & & & & & & & \\
\hline$c r$ & & & $m$ & & & & & & & & & & & & & \\
\hline
\end{tabular}

\begin{tabular}{|c|c|c|c|c|c|c|c|c|c|c|c|c|c|}
\hline 7 & 到 & \begin{tabular}{l|l|l}
31 & 30 \\
\end{tabular} & ${ }^{2}$ & {$\left[{ }_{8}^{\infty}\right]^{3}$} & \begin{tabular}{l|l}
$x_{\mathrm{R}}$ & 26 \\
\end{tabular} & $4^{25} \times 1$ & $e^{24}$ & $2^{23}$ & $x^{2}$ & \begin{tabular}{l|l}
${ }^{x}$ & $K^{x}$ \\
\end{tabular} & $5^{19}$ & $\underline{x}$ & ${ }^{12} x$ \\
\hline R & & MOD & & & & & & & & & 0 & & \\
\hline cc & & & & & & & & & & & $B$ & & \\
\hline$c \mathbf{r}$ & & & & & & & & & & & $m$ & & \\
\hline
\end{tabular}

- Tooth number

Cc - Caries clinical

Ext - Extraction Indicated

R - Restorations present

Cr - Carles radiographic

$X$ - Kising tooth
Rt - Root tip

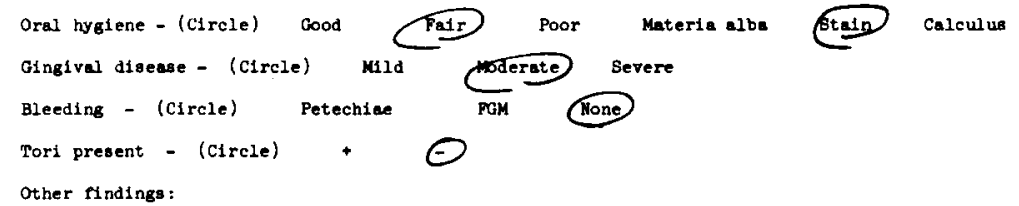

Other flindings:

Treatment needa :

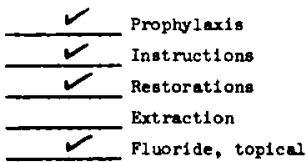

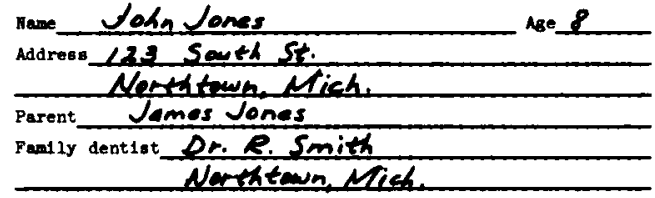

FIGURE 3. Form for recording dental status of hemophiliacs in Michigan. 
potential for spontaneous bleeding and complicated the possibility of conservative dental procedures.

\section{Summer Camp}

Dentists have participated in another form of field clinic activity each year during the summer camp program sponsored by Hemophilia of Michigan. Upon arrival at the camp location, all enrollees received a physical examination and an oral screening examination. The purpose of this dental examination was to identify oral conditions that may potentially erupt as emergent episodes during the week-long camp session. It also provided another opportunity to encourage parents to obtain needed care for their children. It was discouraging to note, however, that some individuals had returned to camp with the same dental problems untreated as were identified the previous summer.

\section{University Combined Specialty Clinic}

In addition to the diagnostic and evaluation clinic activities throughout the state, a similar ongoing evaluation clinic is held at the University of Michigan Medical Center on a biweekly basis. New patients receive hematologic, orthopedic, and dental evaluations. The Director of Social Services of Hemophilia of Michigan also visits with each family to effectively coordinate resources and treatment needs so that the care is obtainable.

The philosophy of this dental care program for hemophiliacs has been one of decentralization. All patients have been encouraged to seek treatment on a regular basis by a dental practitioner in or near their community. This pattern provides for the participation of more professionals and gives assurance to familities that assistance is nearby. Anyone who has been unsuccessful in locating a dentist for treatment is offered such service by the Hemophilia of Michigan office. A list of nearly 200 dentists willing to accept hemophilic patients has been assembled which permits patient referral to a dentist in his area, a general practitioner or a specialist in pedodontics, periodontics, orthodontics, or oral surgery.

Participation in the care of hemophiliacs by Michigan dentists, in addition to their dental school background, is largely a result of education programs and displays at Michigan Dental Association conventions and local dental society meetings, publications in state and national dental journals, and the effectiveness of the field clinics program. Dentists have been informed about the facilities and personnel available to them in times of need at the various treatment centers statewide. Furthermore, at any time Hemophilia Control in Ann Arbor provides phone consultation for management of problems associated with treatment.

Dentists also may choose to refer the patient to the Medical Center in Ann Arbor for diagnosis and treatment. In such cases the emergent and primary needs of the patient are treated and followed by referral to a dentist in his home area to complete the remaining treatment needs. While much has been accomplished, there are challenges to further improve these programs to meet the increasing population of hemophiliacs.

In conclusion, it should be apparent that the medical and dental health 
care team approach is essential, and the effectiveness of the team effort will be enhanced if the following recommendations can be implemented:

1. Since physicians ordinarily are the first to identify hemophiliacs, they are in a strategic position to urge the family to seek and obtain early and regular dental care.

2. Physicians can do much to assist and inform their dentist colleagues in the community regarding hemophilia and his supportive role as treatment is rendered.

3. More dentists must become knowledgeable and confident about their role in the management of patients with hemophilia.

4. The employment of social services personnel by hemophilia organizations to coordinate family needs with treatment availability and sources of support is a vital function of an ongoing program.

5. Development of an effective method is needed to motivate families of hemophiliacs to seek dental care.

6. Preventive dentistry for optimum oral health of persons with hemophilia must be emphasized.

\section{REFERENCES}

1. Webster, W. P., C. W. McMillan, O. N. Lucas \& H. R. Roberts. 1971. Dental management of the bleeder patient. In Proceedings of the VII Congress of the World Federation of Haemophilia, Tehran : 227.

2. Corrigan, J. J. 1972. Oral bleeding in hemophilia: treatment with epsilon aminocaproic acid and replacement therapy. J. Pediat. 80: 124.

3. Megquier, R. J. 1968. Fibrinolytic activity in human dental sockets after extractions. Master's Thesis, Ann Arbor. Univ. of Michigan School of Dentistry. Ann Arbor, Mich.

4. Webster, W. P. \& R. M. Courtney. 1968. Diagnosis and treatment of periodontal disease in the hemophiliac. In Proceedings, Dental Hemophilia Institute. Chapel Hill, N.C.

5. AgeL, D. P. 1968. Psychiatric aspects of hemophilia related to dental care. In Proceedings Dental Hemophilia Institute, Chapel Hill, N.C.

6. Chiono, O. \& O. MiYamoto. 1968. A comprehensive pedodontic program for hemophiliacs. In Proceedings of the Dental Hemophilia Institute, Chapel Hill, N.C.

7. LewIS, B. 1973. Dental care for the hemophiliac. Amer. Dent. A. J. 87: 141115.

8. Snyder, D. T. \& J. A. Penner. 1970. Preventive and restorative dental care for the hemophiliac. Mich. Dent. A. J. 52: 6-8. 